\title{
Lattice Boltzmann model for the simulation of multicomponent mixtures
}

\author{
S. Arcidiacono, ${ }^{1, *}$ I. V. Karlin, ${ }^{2,3, \dagger}$ J. Mantzaras, ${ }^{1, \ddagger}$ and C. E. Frouzakis ${ }^{2, \S}$ \\ ${ }^{1}$ Paul Scherrer Institute, Combustion Research, CH-5232 Villigen PSI, Switzerland \\ ${ }^{2}$ ETH-Zurich, Aerothermochemistry and Combustion Systems Lab, CH-8092 Zurich, Switzerland \\ ${ }^{3}$ School of Engineering Sciences, University of Southampton, SO17 1BJ Southampton, United Kingdom \\ (Received 26 January 2007; revised manuscript received 24 July 2007; published 15 October 2007)
}

\begin{abstract}
A lattice Boltzmann (LB) model for the simulation of realistic multicomponent mixtures is constructed. In the hydrodynamic limit, the LB model recovers the equations of continuum mechanics within the mixtureaveraged diffusion approximation. The present implementation can be used to simulate realistic mixtures with arbitrary Schmidt numbers and molecular masses of the species. The model is applied to the mixing of two opposed jets of different concentrations and the results are in excellent agreement with a continuum model. An application to the simulation of mixtures in microflows is also presented. Results compare well with existing kinetic theory predictions of the slip coefficient for mixtures in a Couette flow.
\end{abstract}

DOI: 10.1103/PhysRevE.76.046703

PACS number(s): 47.11.-j, 05.20.Dd

\section{INTRODUCTION}

Realistic simulation of gas mixtures is important in many areas of science and engineering. The recent revival of interest to computational kinetic theory stems in part from the development of micro electro-mechanical systems (MEMS) and vacuum technology, as well as energy convertors such as solid oxide fuel cells (SOFCs). In such problems, the traditional continuous description of mass and momentum transport breaks down when the mean free path becomes comparable to a characteristic size of the system with the increase of rarefaction, and approaches based on kinetic theory are sought.

It is well known that the computationally-oriented kinetic theory of mixtures is notoriously difficult and lags substantially behind the single-component case. On the one hand, standard approaches based on molecular dynamics or direct simulation Monte Carlo (DSMC) become increasingly expensive as the continuum limit is approached. On the other hand, approaches based on the classical Boltzmann equation or kinetic models thereof are difficult to apply to mixtures beyond stationary linearized problems.

Recently, the lattice Boltzmann (LB) model provided an alternative to conventional macroscopic models and many attempts have been made to extend this approach to the simulation of mixtures [1-17]. The LB method is also appealing for simulating microflows [18-33], and for its simple algorithm that can be easily modified for parallel computing. However, most of the proposed LB models for mixtures have a number of drawbacks. The single-fluid approach [using only one Bhatnagar-Gross-Krook (BGK) collision term to describe the average properties of the mixture] proved to be

\footnotetext{
*salvatore.arcidiacono@psi.ch

† Author to whom correspondence should be addressed. karlin.ilya@gmail.com

*ioannis.mantzaras@psi.ch

${ }^{\S}$ frouzakis@lav.mavt.ethz.ch
}

accurate for certain problems, but it has limitations in the mesoscopic description and shows numerical instabilities when the density variation of the mixture is significant. A much more amenable class of models is based on the multiple-fluid approach that can include more physics in the system description. In this kind of approach, each species is relaxing to equilibrium with different relaxation times, simulating self- and cross-collisions. However, most of the models introduced in the past do not satisfy important physical properties such as the $H$ theorem (it is not ensured that the entropy production is non-negative) or the indifferentiability principle (they do not reduce to the single-component BGK fluid when the species become mechanically equivalent), and face numerical instabilities at large molecular mass ratios. Moreover, the proposed multiple-fluid models have not yet been applied to realistic systems.

In the present work, the recently introduced LB model for of binary mixtures $[34,35]$ is extended to the simulation of multicomponent mixtures of arbitrary Schmidt numbers Sc. The model is based on the fast-slow decomposition of motions near a quasiequilibrium $(\mathrm{QE})$ manifold, with each species distribution approaching equilibrium in two steps: first from the initial state to the $\mathrm{QE}$ and then from the $\mathrm{QE}$ to equilibrium. Two corresponding relaxation times are used. In order to simulate mixtures at any Schmidt number and, at the same time, to satisfy the $H$ theorem, two different QE distribution functions are required. The model ensures (i) thermodynamic consistency (the $H$ theorem is satisfied), (ii) the indifferentiability principle, (iii) the recovery of the NavierStokes equations and of the Stefan-Maxwell diffusion equations (within the macroscopic mixture-averaged approximation $[36,37]$ ) in the hydrodynamic limit, and (iv) the capability to simulate large molecular weight ratios. The model has been implemented in a code that allows for the computation of realistic mixture transport properties (viscosity and binary diffusion coefficients) at each time step and at each grid node by employing the CHEMKIN transport package [38].

The model and its implementation are validated in two problems covering different domains of applicability. In the macroscopic regime, we simulated the mixing of two streams of different concentrations issued from two planar opposing 
jets. In the microscopic regime, we evaluated the slip coefficient of a gas mixture between two parallel plates (Couette flow). Results are in good agreement with a fluid dynamics solver in the continuum regime, and with the DSMC and a solution to the kinetic equation in the microflow regime, respectively.

The paper is organized as follows. The theoretical background of the model is described in Secs. II-V. The numerical implementation is reported in Sec. VI and applications are presented in Sec. VII. Conclusions are finally given in Sec. VIII.

\section{KINETIC MODEL FOR MULTICOMPONENT MIXTURES}

The proposed kinetic model is based on a representation of the fast-slow decomposition of motions near a QE state $[39,40]$. In such a representation, the relaxation from an initial state represented by the distribution function $f$ to the equilibrium $f^{\text {eq }}$ is decomposed into two steps: a "fast" motion from $f$ to the quasiequilibrium state $f^{*}$ and a "slow" motion from $f^{*}$ to $f^{\text {eq }}$. Both motions can be approximated by a BGK term with the condition $\tau_{2} \geqslant \tau_{1}$, where $\tau_{1}$ and $\tau_{2}$ are the relaxation times from $f$ to $f^{*}$ and from $f^{*}$ to $f^{\text {eq }}$, respectively.

Exploiting this fast-slow motion decomposition, the generic discrete-velocity kinetic equation for each species $j$ in a mixture with $M$ components can be written as

$$
\begin{aligned}
\partial_{t} f_{j i}+c_{j i \alpha} \partial_{\alpha} f_{j i}= & -\frac{1}{\tau_{1 j}}\left(f_{j i}-f_{j i}^{*}\right)-\frac{1}{\tau_{2 j}}\left(f_{j i}^{*}-f_{j i}^{\mathrm{eq}}\right) \\
& +F_{j i}, \quad j=1, \ldots, M, \quad i=0, \ldots, N,
\end{aligned}
$$

where $N$ is the number of the discrete lattice velocities $c_{j i \alpha}$, $\alpha=\{x, y\}$ is the spacial direction, $\tau_{1 j}$ and $\tau_{2 j}$ are the relaxation times of each species $j$ from the initial state to the QE and

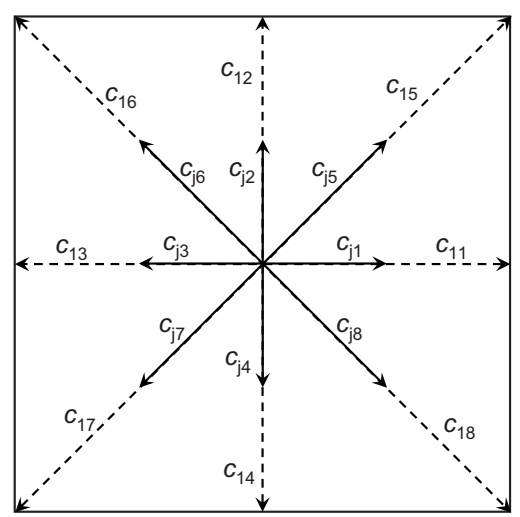

FIG. 1. Discrete velocity vectors of various species. The velocities $c_{1 i}$ of the lighter component of mass $m_{1}$ are on the lattice. The corresponding magnitudes are $c_{1\{1,2,3,4\}}=c_{1}$ and $c_{1\{5,6,7,8\}}=c_{1} \sqrt{2}$. The generic off-lattice velocity vectors $c_{j i}$ corresponding to species with the molecular mass $m_{j}>m_{1}$ are also represented.

from the $\mathrm{QE}$ to equilibrium, respectively, and $F_{j i}$ is a forcing term acting on species $j$ that will be defined below. In Eq. (1) and subsequently, Einstein's summation convention is applied only to the spacial direction $\alpha$.

In order to evaluate the $\mathrm{QE}$ and the equilibrium distribution functions, the $H$ function is defined according to Ref. [41]:

$$
H=\sum_{j}^{M} \sum_{i}^{N} f_{j i} \ln \frac{f_{j i}}{W_{i}}
$$

where $W_{i}$ are the weights associated to the corresponding discrete lattice velocities $c_{j i}$ [42]. For the D2Q9 implementation considered herein (Fig. 1): and

$$
W_{i}= \begin{cases}\frac{4}{9}, & i=0 \\ \frac{1}{9}, & i=1 \text { to } 4 \\ \frac{1}{36}, & i=5 \text { to } 8 .\end{cases}
$$

In Eq. (3), the magnitudes $c_{j}$ of the discrete velocities $c_{j i}$ are related to the speed of sound $c_{s j}=\sqrt{R T_{0} / m_{j}}$ of each species as $c_{j}=\sqrt{3} c_{s j}$ (see Fig. 1), where $m_{j}$ is the molecular mass of component $j, R$ is the ideal gas constant, and $T_{0}$ is a reference temperature. Pertinent moments of the populations are defined as follows:

$$
\rho_{j}=\sum_{i}^{N} f_{j i}, \quad J_{j \alpha}=\sum_{i}^{N} f_{j i} c_{j i \alpha},
$$




$$
P_{j \alpha \beta}=\sum_{i}^{N} f_{j i} c_{j i \alpha} c_{j i \beta}, \quad Q_{j \alpha \beta \gamma}=\sum_{i}^{N} f_{j i} c_{j i \alpha} c_{j i \beta} c_{j i \gamma}
$$

where $\rho_{j}, J_{j \alpha}, P_{j \alpha \beta}$, and $Q_{j \alpha \beta \gamma}$ are the density, the momentum, the pressure tensor, and the third-order moment of component $j$, respectively.

The equilibrium distribution function is evaluated by minimizing Eq. (2) under the constraints for conservation of the density of each species $\left(\rho_{j}\right)$, and of the momentum of the mixture $\mathbf{J}=\Sigma_{j}^{M} \mathbf{J}_{j}$ (energy conservation is not relevant in the present isothermal model):

$$
\rho_{j}=\sum_{i}^{N} f_{j i}, \quad J_{\alpha}=\sum_{j}^{M} \sum_{i}^{N} f_{j i} c_{j i \alpha} .
$$

The result of the minimization problem can be directly extracted from the equilibrium of a single-component fluid [42]

$$
\begin{aligned}
f_{j i}^{\mathrm{eq}}\left(\rho_{j}, \mathbf{U}\right)= & \rho_{j} W_{i} \prod_{\alpha=1}^{d}\left(\frac{2 c_{j}-\sqrt{c_{j}^{2}+3 U_{\alpha}^{2}}}{c_{j}}\right) \\
& \times\left(\frac{2 U_{\alpha}+\sqrt{c_{j}^{2}+3 U_{\alpha}^{2}}}{c_{j}-U_{\alpha}}\right)^{c_{j i \alpha} / c_{j}},
\end{aligned}
$$

where $d$ is the spatial dimension of the system, $U_{\alpha}=J_{\alpha} / \rho$ is the velocity of the mixture in the $\alpha$ direction, and $\rho=\sum_{j}^{M} \rho_{j}$ is the mixture density.

The QE distribution function $f_{j}^{*}$ of each species $j$ is obtained by minimizing the $H$ function under the same constraints of the conserved variables and some additional linear constraints on "quasiconserved" variables that are considered to approach the equilibrium slowly, while all other moments are assumed to equilibrate faster. The quasiconserved variable can be chosen by examining the parameters affecting the particular problem. An indicator of the effectiveness of mass transport due to diffusion and momentum for each species $j$ is the Schmidt number $S c_{j}=\nu / D_{j m}$ ( $\nu$ is the kinematic viscosity and $D_{j m}$ a mixture-averaged diffusion coefficient for species $j$ that will be defined in Sec. III A). The moments related to these two transport processes are (1) the pressure tensor $P_{j \alpha \beta}$ of species $j$ and (2) the difference between the momentum $\mathbf{J}$ of the mixture and the momentum $\mathbf{J}_{j}$ of species $j$, respectively. Both moments 1 and 2 can serve as slow variables of the problem and the particular choice of one over the other is dictated by the condition $\tau_{1 j} \leqslant \tau_{2 j}$ that ensures the $H$ theorem and thus affects the numerical stability of the computational scheme. It will be demonstrated below that this condition results in the definition of a reference Schmidt number $\mathrm{Sc}_{j}{ }^{*}$ that in turn leads to two complementary models that can simulate mixtures with species obeying either $\mathrm{Sc}_{j} \geqslant \mathrm{Sc}_{j}^{*}$ (Sec. III) or $\mathrm{Sc}_{j} \leqslant \mathrm{Sc}_{j}^{*}$ (Sec. IV), depending on the slow variable of the problem. In a mixture with more than two species a third option exists, where the momentum difference is the slow variable for some species, while for the rest it is the pressure tensor $P_{j \alpha \beta}$. In this case both models are used locally, as discussed in Sec. V.

The first QE is obtained by minimizing the $H$ function using the linear constraint 1 . The constraints for this case can be written as

$$
\begin{gathered}
\rho_{j}=\sum_{i}^{N} f_{j i}, \\
J_{\alpha}=\sum_{j}^{M} \sum_{i}^{N} f_{j i} c_{j i \alpha}, \\
P_{j \alpha \beta}=\sum_{i}^{N} f_{j i} c_{j i \alpha} c_{j i \beta} .
\end{gathered}
$$

For this minimization problem it is not possible to find an analytical expression directly and some approximations are needed. In the vicinity of equilibrium, a solution can be obtained by expanding the $H$ function around the equilibrium (triangle entropy method) $[39,43,44]$, and the QE can be expressed as

$$
\begin{aligned}
f_{j i}^{*}\left(\rho_{j}, J_{\alpha}, P_{j \alpha \beta}\right)= & f_{j i}^{\mathrm{eq}}\left(1+\chi+\beta_{x} c_{i x}+\beta_{y} c_{i y}+\gamma_{x x} c_{i x} c_{i x}\right. \\
& \left.+\gamma_{y y} c_{i y} c_{i y}+\gamma_{x y} c_{i x} c_{i y}\right),
\end{aligned}
$$

where $\chi, \beta_{x}, \beta_{y}, \gamma_{x x}, \gamma_{y y}, \gamma_{x y}$ are the Lagrange multipliers reported in the Appendix.

Equation (9) involves the evaluation of many polynomials and can be computationally expensive. A simpler expression of the QE distribution function can be obtained by noting that Grad's distribution [45]

$$
\begin{aligned}
f_{j i}^{*}\left(\rho_{j}, J_{\alpha}, P_{j \alpha \beta}\right)= & W_{i}\left[\rho_{j}+\frac{J_{\alpha} c_{j i \alpha}}{c_{j s}^{2}}+\frac{1}{2 c_{j s}^{4}}\left(P_{j \alpha \beta}-\rho_{j} c_{j s}^{2} \delta_{\alpha \beta}\right)\right. \\
& \left.\times\left(c_{j i \alpha} c_{j i \beta}-c_{j s}^{2} \delta_{\alpha \beta}\right)\right]
\end{aligned}
$$

also satisfies the consistency relations (8), as the QE itself, and thus ensures the conservation of mass, momentum, and pressure in the fast relaxation. It is noted that the use of Eq. (10) to represent the QE is computationally more efficient, but the $H$ theorem (thermodynamic consistency) is not ensured anymore. Nonetheless, in all the simulations presented herein no stability issues where observed when such distribution was used. Moreover, no appreciable difference was noticed in the results when using either Eq. (9) or (10).

The second QE is derived using the quasi-conserved variable 2, as already discussed in detail in Refs. [34,35]. A new set of constraints for the minimization of the $H$ function is then obtained:

$$
\begin{gathered}
\rho_{j}=\sum_{i}^{N} f_{j i}, \\
J_{\alpha}=\sum_{j}^{M} \sum_{i}^{N} f_{j i} c_{j i \alpha}, \\
J_{\alpha}-J_{j \alpha}=\sum_{j} \sum_{i}^{N} f_{j i} c_{j i \alpha}-\sum_{i}^{N} f_{j i} c_{j i \alpha},
\end{gathered}
$$

which is equivalent to 


$$
\begin{gathered}
\rho_{j}=\sum_{i}^{N} f_{j i}, \\
J_{j \alpha}=\sum_{i}^{N} f_{j i} c_{j i \alpha} .
\end{gathered}
$$

It can be easily shown that the minimization problem is the same as in the single-component case, and that the QE populations are obtained by substituting in Eq. (7) the velocity of each component $\mathbf{U}_{j}=\mathbf{J}_{j} / \rho_{j}$ instead of the total mixture velocity

$$
f_{j i}^{*}\left(\rho_{j}, \mathbf{U}_{j}\right)=f_{j i}^{\mathrm{eq}}\left(\rho_{j}, \mathbf{U}_{j}\right) .
$$

It is finally noted that for both $\mathrm{QE}$ the indifferentiability principle is satisfied when all the molecular weights $m_{j}$ are the same, and $\tau_{1 j}=\tau_{2 j}=\tau$, where $\tau$ is a constant for $j$ $=1, \ldots, M[$ Eq. (1)].

Thus, we have identified the QE populations (or approximations thereof) for both cases 1 and 2 mentioned above. Now we proceed with an analysis of the hydrodynamic limit of the corresponding kinetic Eqs. (1) for each case separately.

\section{MODEL I: PRESSURE AS SLOW VARIABLE}

We begin by investigating the hydrodynamic limit of the kinetics Eqs. (1) for case 1, i.e., $f_{j i}^{*}=f_{j i}^{*}\left(\rho_{j}, J_{\alpha}, P_{j \alpha \beta}\right)$. Multiplying Eqs. (1) by $\left[1, c_{j i \alpha}, c_{j i \alpha} c_{j i \beta}\right]$, summing up and further using Eqs. (5), the moment transport equations for each species $j$ are obtained.

Individual density:

$$
\partial_{t} \rho_{j}+\partial_{\alpha} J_{j \alpha}=0
$$

Individual momentum:

$$
\partial_{t} J_{j \alpha}+\partial_{\beta} P_{j \alpha \beta}=\frac{1}{\tau_{1 j}}\left(\frac{\rho_{j}}{\rho} J_{\alpha}-J_{j \alpha}\right) .
$$

Individual pressure tensor:

$$
\partial_{t} P_{j \alpha \beta}+\partial_{\gamma} Q_{j \alpha \beta \gamma}=\frac{1}{\tau_{2 j}}\left(P_{j \alpha \beta}^{\mathrm{eq}}-P_{j \alpha \beta}\right),
$$

with $P_{j \alpha \beta}^{\mathrm{eq}}$ the pressure tensor of component $j$ at equilibrium. The total momentum of the mixture is given by summing up Eqs. (15):

$$
\partial_{t} J_{\alpha}+\partial_{\beta} P_{\alpha \beta}=\sum_{j}^{M} \frac{1}{\tau_{1 j}}\left(\frac{\rho_{j}}{\rho} J_{\alpha}-J_{j \alpha}\right),
$$

where $P_{\alpha \beta}=\sum_{j}^{M} P_{j \alpha \beta}$ is the mixture pressure tensor. The righthand side of Eq. (17) vanishes, i.e., the mixture momentum is locally conserved, if and only if

$$
\sum_{j}^{M} \frac{1}{\tau_{1 j}}\left(\frac{\rho_{j}}{\rho} J_{\alpha}-J_{j \alpha}\right)=-\sum_{j}^{M} \frac{\rho_{j} V_{j \alpha}}{\tau_{1 j}}=0,
$$

where $V_{j \alpha}$ is the diffusion velocity of species $j$ in the direction $\alpha$, defined as

$$
\rho_{j} V_{j \alpha}=-\left(\frac{\rho_{j}}{\rho} J_{\alpha}-J_{j \alpha}\right) .
$$

The conditions under which Eq. (18) is satisfied will be discussed at the end of Sec. III A. By the definition of Eq. (19), the following equality is also satisfied:

$$
\sum_{j}^{M} \rho_{j} V_{j \alpha}=0
$$

Using the standard Chapman-Enskog expansion, it is possible to recover the viscosity and diffusion coefficients in the hydrodynamic limit from Eqs. (15) and (16) by expanding all nonconserved variables and time derivatives around the equilibrium in terms of the Kundsen number

$$
\begin{gathered}
\partial_{t}=\partial_{t}^{(0)}+\mathrm{Kn} \partial_{t}^{(1)}+\mathrm{Kn}^{2} \partial_{t}^{(2)}+\cdots, \\
P_{\alpha \beta}=P_{\alpha \beta}^{\mathrm{eq}}+\mathrm{Kn} P_{\alpha \beta}^{(1)}+\cdots, \\
V_{j \alpha}=V_{j \alpha}^{\mathrm{eq}}+\mathrm{Kn} V_{j \alpha}^{(1)}=\mathrm{Kn} V_{j \alpha}^{(1)}+\cdots .
\end{gathered}
$$

The diffusion velocity $V_{j \alpha}^{\mathrm{eq}}$ is equal to zero, since at the local equilibrium all the components have the same velocity. The expansion is carried out with the further assumption that $\tau_{1 j}$ and $\tau_{2 j}$ are of $O(\mathrm{Kn})$. For the present model, the equilibrium pressure tensor and third-order moment of component $j$ are

$$
\begin{gathered}
P_{j \alpha \beta}^{\mathrm{eq}}=n_{j} R T_{0} \delta_{\alpha \beta}+\frac{\rho_{j}}{\rho} \frac{J_{\alpha} J_{\beta}}{\rho}, \\
Q_{j \alpha \beta \gamma}^{\mathrm{eq}}=n_{j}\left(\frac{J_{\alpha}}{\rho} \delta_{\beta \gamma}+\frac{J_{\beta}}{\rho} \delta_{\alpha \gamma}+\frac{J_{\gamma}}{\rho} \delta_{\alpha \beta}\right) R T_{0},
\end{gathered}
$$

where $n_{j}=\rho_{j} / m_{j}$ is the concentration (moles per unit volume) of species $j$. As pertinent to the zeroth order time derivatives, the conserved moments become

$$
\begin{gathered}
\partial_{t}^{(0)} \rho_{j}=\partial_{\alpha}\left(\frac{\rho_{j}}{\rho} J_{\alpha}\right), \\
\partial_{t}^{(0)} J_{\alpha}=-\partial_{\beta} P_{\alpha \beta}^{\mathrm{eq}} .
\end{gathered}
$$

\section{A. Diffusion coefficient}

The diffusion transport equation for species $j$ can be obtained from the single component momentum Eq. (15). Using Eqs. (22) and (23), the time dependence in Eq. (15) can be eliminated. After some algebra, Eq. (15) becomes

$$
\partial_{\alpha} X_{j}=-\frac{\rho_{j} V_{j \alpha}}{P \tau_{1 j}}+\left(Y_{j}-X_{j}\right) \frac{\partial_{\alpha} P}{P},
$$

where $P=n R T_{0}$ is the ideal gas mixture pressure, $X_{j}$ and $Y_{j}$ are the molar and the mass fraction of species $j$, respectively, and $n=\sum_{j}^{M} n_{j}$ are the total number of moles per unit volume. The relaxation time $\tau_{1 j}$ is obtained by comparing Eq. (24) with its macroscopic counterpart, the Stefan-Maxwell diffusion equation for an isothermal system in the absence of body forces $[36,37]$ 


$$
\partial_{\alpha} X_{j}=\sum_{k \neq j}^{M} \frac{X_{j} X_{k}}{\mathcal{D}_{j k}}\left(\frac{J_{j \alpha}}{\rho_{j}}-\frac{J_{k \alpha}}{\rho_{k}}\right)+\left(Y_{j}-X_{j}\right) \frac{\partial_{\alpha} P}{P},
$$

where $\mathcal{D}_{j k}$ is the binary diffusion coefficient between components $j$ and $k$. Rewriting the diffusion velocity as

$$
\begin{aligned}
\rho_{j} V_{j \alpha} & =J_{j \alpha}-\frac{\rho_{j}}{\rho} J_{\alpha} \\
& =\frac{1}{\rho}\left(J_{j \alpha} \sum_{k \neq j}^{M} \rho_{k}-\rho_{j} \sum_{k \neq j}^{M} J_{k \alpha}\right)=\frac{1}{\rho_{k \neq j}} \sum_{k \neq j}^{M}\left(J_{j \alpha} \rho_{k}-\rho_{j} J_{k \alpha}\right) \\
& =\sum_{k \neq j}^{M}\left(\frac{J_{j \alpha}}{\rho_{j}}-\frac{J_{k \alpha}}{\rho_{k}}\right) \frac{\rho_{j} \rho_{k}}{\rho}
\end{aligned}
$$

substituting it into Eq. (24), and comparing Eq. (24) with Eq. (25), we find

$$
\tau_{1 j}=\frac{\sum_{k \neq j}^{M} \frac{\rho_{j} \rho_{k}}{\rho}\left(\frac{J_{j \alpha}}{\rho_{j}}-\frac{J_{k \alpha}}{\rho_{k}}\right)}{P \sum_{k \neq j}^{M} \frac{X_{j} X_{k}}{\mathcal{D}_{j k}}\left(\frac{J_{j \alpha}}{\rho_{j}}-\frac{J_{k \alpha}}{\rho_{k}}\right)} .
$$

Equation (27) is an exact expression, but, contrary to the binary mixture case, the relaxation time $\tau_{1 j}$ depends on the velocities as well as on the spacial direction $\alpha$. This problem stems from the fact that $\tau_{1 j}$ is a $M$-component vector which cannot completely represent the $M \times M$ matrix of binary diffusion coefficients $\mathcal{D}_{j k}$. Since it is preferable to have the relaxation time depending only on fluid local transport properties and not on the flow, an approximation is required. A common approach is the so-called mixture-averaged diffusion approximation [36,37]. Assuming initially that the quantity $\left(J_{j \alpha} / \rho_{j}-J_{k \alpha} / \rho_{k}\right)$ is of the same order for all $j \neq k$, an average diffusion coefficient for species $j$ is obtained. In a second step, an appropriate correction term will be applied to remove the introduced error. With this, we obtain in Eq. (27):

$$
\tau_{1 j}=\frac{\sum_{k \neq j}^{M} \rho_{j} \rho_{k} / \rho}{P \sum_{k \neq j}^{M} X_{j} X_{k} / \mathcal{D}_{j k}}=\frac{\rho_{j}}{P X_{j}} \frac{1-Y_{j}}{\sum_{k \neq j}^{M} X_{k} / \mathcal{D}_{j k}}=\frac{\rho_{j}}{P X_{j}} D_{j m},
$$

where

$$
D_{j m}=\frac{1-Y_{j}}{\sum_{k \neq j}^{M} X_{k} / \mathcal{D}_{j k}},
$$

is the mixture-averaged diffusion coefficient of component $j$. Note that the mixture-averaged diffusion coefficient obtained from the QE model is the same as the one used in other mixture-averaged models of fluid dynamics (see, for example, Ref. [38]). This formulation is widely used, for example, in combustion and it is correct asymptotically when there is either only one bulk component and $M-1$ trace species, or when all species but one have nearly the same diffu- sion velocities, or when all the diffusion coefficients are the same. In the limit of a binary mixture, the relation reported in Refs. [34,35] is recovered either from Eq. (27) or Eq. (28):

$$
\tau_{11}=\tau_{12}=\frac{\mu_{12} D}{P X_{1} X_{2}},
$$

where $\mu_{12}=\rho_{1} \rho_{2} /\left(\rho_{1}+\rho_{2}\right)$ is the reduced mass, and $D=\mathcal{D}_{12}$ $=\mathcal{D}_{21}$.

The requirement of momentum conservation Eq. (18) can now be written, with the aid of Eqs. (24) and (25), as

$$
\sum_{j}^{M} \frac{\rho_{j} V_{j \alpha}}{P \tau_{1 j}}=\sum_{j}^{M} \sum_{k}^{M} \frac{X_{j} X_{k}}{\mathcal{D}_{j k}}\left(\frac{J_{j \alpha}}{\rho_{j}}-\frac{J_{k \alpha}}{\rho_{k}}\right),
$$

which vanishes when $\mathcal{D}_{j k}=\mathcal{D}_{k j}$, i.e., when the binary diffusion matrix is symmetric. Considering that $\tau_{1 j}$ was defined by using the mixture-averaged approximation (28), the momentum conservation (18) can no longer be satisfied. In order to enforce momentum conservation, a correction velocity $V_{c \alpha}$ is usually introduced [36,37]:

$$
J_{j \alpha}=\widetilde{J}_{j \alpha}+\rho_{j} V_{c \alpha}
$$

where $\widetilde{j}_{j}$ is the momentum of species $j$. By imposing that Eq. (18) is satisfied, the correction velocity is

$$
\sum_{j}^{M} \frac{\rho_{j}}{\tau_{1 j}} V_{c \alpha}=-\sum_{j}^{M} \frac{\frac{\rho_{j}}{\rho} J_{\alpha}-\widetilde{J}_{j \alpha}}{\tau_{1 j}} .
$$

In order to apply this velocity correction, a forcing term is added to Eq. (1):

$$
F_{V j i \alpha}=\psi_{j i \alpha} \frac{\rho_{j} V_{c \alpha}}{\tau_{1 j}},
$$

where $\psi_{j i \alpha}$ are weights acting only on the momentum equation, i.e.,

$$
\sum_{i}^{N} c_{j i \alpha} \psi_{j i \alpha}=1
$$

while the contribution to all the other moments is set to zero.

\section{B. Viscosity}

In analogy with the diffusion coefficient, Eqs. (16) allow for the evaluation of the shear viscosity. Using the ChapmanEnskog procedure again, after some rearrangement Eqs. (16) lead to

$$
P_{j \alpha \beta}^{\mathrm{eq}}-P_{j \alpha \beta}=\tau_{2 j} n_{j} R T_{0}\left(\partial_{\alpha} \frac{J_{\beta}}{\rho}+\partial_{\beta} \frac{J_{\alpha}}{\rho}\right)
$$

or, upon summation over the species,

$$
P_{\alpha \beta}=P_{\alpha \beta}^{\mathrm{eq}}-R T_{0}\left(\partial_{\alpha} \frac{J_{\beta}}{\rho}+\partial_{\beta} \frac{J_{\alpha}}{\rho}\right) \sum_{j}^{M} \tau_{2 j} n_{j} .
$$

Substituting the latter expression into Eq. (17) and comparing it with the Navier-Stokes equations 


$$
\partial_{t} J_{\alpha}+\partial_{\alpha} P+\partial_{\beta}\left[\frac{J_{\alpha} J_{\beta}}{\rho}-\mu\left(\partial_{\alpha} \frac{J_{\beta}}{\rho}+\partial_{\beta} \frac{J_{\alpha}}{\rho}\right)\right]=0,
$$

the viscosity coefficient is immediately identified as

$$
\mu=R T_{0} \sum_{j}^{M} \tau_{2 j} n_{j}
$$

In a multicomponent mixture, the viscosity is a complicated function of the concentrations of different components $[36,37]$. In the present implementation it is assumed that the viscosity obeys the Wilke formula [46] modified by Bird et al. [36]:

$$
\mu=\sum_{j}^{M} \frac{X_{j} \mu_{j}}{\sum_{k}^{M} X_{k} \Phi_{j k}},
$$

where $\mu_{j}$ is the viscosity of the single component $j$ and

$$
\Phi_{j k}=\frac{1}{\sqrt{8}}\left(1+\frac{m_{j}}{m_{k}}\right)^{-1 / 2}\left[1+\left(\frac{\mu_{j}}{\mu_{k}}\right)^{1 / 2}\left(\frac{m_{k}}{m_{j}}\right)^{1 / 4}\right]^{2} .
$$

Comparing Eqs. (39) and (40), we identify the relaxation times $\tau_{2 j}$ in terms of the viscosity coefficients

$$
\tau_{2 j}=\frac{\mu_{j}}{P \sum_{k} X_{k} \Phi_{j k}}=\frac{\mu_{j \mathrm{eff}}}{P} .
$$

The relaxation times $\tau_{2 j}$ are thus depending on the "effective" viscosity $\mu_{\text {jeff }}$ of component $j$ in the mixture.

Now that the diffusion coefficients and the viscosity of the mixture have been identified, the implication of the fast-slow decomposition with its requirement $\tau_{1 j} \leqslant \tau_{2 j}$ can be studied. We define the Schmidt number of component $j$ as

$$
\mathrm{Sc}_{j}=\frac{\mu_{\text {eff }}}{\rho D_{j m}}=\frac{\tau_{2 j}}{\tau_{1 j}} \frac{Y_{j}}{X_{j}} \geqslant \frac{Y_{j}}{X_{j}} \equiv \mathrm{Sc}_{j}^{*},
$$

which implies that locally for the model I derived above, the Schmidt number of each component has to be larger than the reference quantity $\mathrm{Sc}_{j}^{*}$. The latter is only a function of the local concentration of species $j$.

\section{MODEL II: MOMENTUM DIFFERENCE AS SLOW VARIABLE}

Assuming now that the slow variable is the momentum difference, i.e., $f^{*}=f^{*}\left(\rho_{j}, \mathbf{U}_{j}\right)$, and evaluating the moment transport equations as done above for model I, the equations for the conserved moments Eqs. (14) and (17) are obtained again (as expected), while the balance equations for the nonconserved moments read as follows.

Individual momentum:

$$
\partial_{t} J_{j \alpha}+\partial_{\beta} P_{j \alpha \beta}=\frac{1}{\tau_{2 j}}\left(\frac{\rho_{j}}{\rho} J_{\alpha}-J_{j \alpha}\right) .
$$

Individual pressure:

$$
\begin{aligned}
\partial_{t} P_{j \alpha \beta}+\partial_{\gamma} Q_{j \alpha \beta \gamma}= & \frac{1}{\tau_{1 j}}\left(P_{j \alpha \beta}^{*}-P_{j \alpha \beta}\right)+\frac{1}{\tau_{2 j}}\left(P_{j \alpha \beta}^{\mathrm{eq}}-P_{j \alpha \beta}^{*}\right) \\
= & \frac{1}{\tau_{1 j}}\left(P_{j \alpha \beta}^{\mathrm{eq}}-P_{j \alpha \beta}\right)+\left(\frac{1}{\tau_{2 j}}-\frac{1}{\tau_{1 j}}\right) \\
& \times\left(P_{j \alpha \beta}^{\mathrm{eq}}-P_{j \alpha \beta}^{*}\right) .
\end{aligned}
$$

The second term in the right-hand side of Eq. (45) is a spurious term that is not summing up to zero when recovering the total pressure equation unless $\tau_{1 j}$ are the same for all components [35]. In order to eliminate this problem, a counterterm has to be introduced in Eq. (1) such that this contribution is canceled:

$$
F_{P j i \alpha \beta}=-\Psi_{j i \alpha \beta}\left(\frac{1}{\tau_{2 j}}-\frac{1}{\tau_{1 j}}\right)\left(P_{j \alpha \beta}^{\mathrm{eq}}-P_{j \alpha \beta}^{*}\right),
$$

where $\Psi_{j i \alpha \beta}$ are weights that are applied only to the pressure equation, i.e.,

$$
\sum_{i}^{N} c_{j i \alpha} c_{j i \beta} \Psi_{j i \alpha \beta}=1
$$

while the other moments of $\Psi_{j i \alpha \beta}$ are set to zero. Through the Chapman-Enskog expansion the viscosity and diffusion coefficient are recovered:

$$
\begin{gathered}
\tau_{1 j}=\frac{\mu_{j \mathrm{eff}}}{P}, \\
\tau_{2 j}=\frac{\rho_{j}}{P X_{j}} D_{j m} .
\end{gathered}
$$

Finally, considering the fast-slow decomposition condition $\tau_{1 j} \leqslant \tau_{2 j}$, the restriction on the Schmidt number is

$$
\mathrm{Sc}_{j}=\frac{\mu}{\rho D_{j m}}=\frac{\tau_{1 j}}{\tau_{2 j}} \frac{Y_{j}}{X_{j}} \leqslant \frac{Y_{j}}{X_{j}} \equiv \mathrm{Sc}_{j}^{*} .
$$

Now the inequality direction is reversed compared to Eq. (43), showing that the model II is complementary to model I. If the inequality $\mathrm{Sc}_{j} \geqslant \mathrm{Sc}_{j}{ }_{j}$ is satisfied for all the components in a flow, model I will be used. On the other hand, model II is used when $\mathrm{Sc}_{j} \leqslant \mathrm{Sc}_{j}^{*}$ for all components. In practical applications it is possible that some components satisfy the first inequality [Eq. (43)] while the rest satisfy the second [Eq. (50)]. In such cases, the use of the two quasiequilibria locally at the same time is required. The joint implementation of the two models is straightforward (see next section).

\section{COMBINATION OF TWO MODELS}

Denoting $m$ as the number of components for which the QE model I must be used, the moment equations for the system can be written as

$$
\begin{gathered}
\partial_{t} \rho_{j}+\partial_{\alpha} J_{j \alpha}=0 \\
\partial_{t} J_{\alpha}+\partial_{\beta} P_{\alpha \beta}=\sum_{j}^{m} \frac{1}{\tau_{1 j}^{\mathrm{I}}}\left(\frac{\rho_{j}}{\rho} J_{\alpha}-J_{j \alpha}\right)+\sum_{j}^{M-m} \frac{1}{\tau_{2 j}^{\mathrm{II}}}\left(\frac{\rho_{j}}{\rho} J_{\alpha}-J_{j \alpha}\right),
\end{gathered}
$$




$$
\begin{aligned}
\partial_{t} P_{j \alpha \beta}+\partial_{\gamma} Q_{j \alpha \beta \gamma}= & \sum_{j}^{m} \frac{1}{\tau_{2 j}^{\mathrm{I}}}\left(P_{j \alpha \beta}^{\mathrm{eq}}-P_{j \alpha \beta}\right) \\
& +\sum_{j}^{M-m} \frac{1}{\tau_{1 j}^{\mathrm{II}}}\left(P_{j \alpha \beta}^{\mathrm{eq}}-P_{j \alpha \beta}\right),
\end{aligned}
$$

where the superscripts I, II denote model I or II, respectively. Taking into account that $\tau_{2 j}^{\mathrm{I}}$ and $\tau_{1 j}^{\mathrm{II}}$ are calculated from the viscosities whereas $\tau_{1 j}^{\mathrm{I}}$ and $\tau_{2 j}^{\mathrm{II}}$ are evaluated from the diffusion coefficients, Eqs. (51) imply that when the two models are used together all the mixture moment transport equations are recovered correctly.

\section{NUMERICAL IMPLEMENTATION}

Equations (1) are discretized in time by applying the implicit trapezoidal rule between time $t$ and $t+\delta t$ :

$$
\begin{aligned}
f_{j i}\left(\mathbf{x}+c_{j i} \delta t, t+\delta t\right)= & f_{j i}(\mathbf{x}, t)+\frac{\delta t}{2}\left\{\Omega_{j i}[f(\mathbf{x}, t)]\right. \\
& \left.+\Omega_{j i}\left[f\left(\mathbf{x}+c_{j i} \delta t, t+\delta t\right)\right]\right]+O\left(\delta t^{3}\right),
\end{aligned}
$$

with $\Omega_{j i}[f(\mathbf{x}, t)]$ the collision integral at time $t$. This scheme is rendered explicit by introducing a local transformation through the auxiliary functions $g_{j i}$ :

$$
g_{j i}(\mathbf{x}, t)=f_{j i}(\mathbf{x}, t)-\frac{\delta t}{2} \Omega_{j i}[f(\mathbf{x}, t)] .
$$

Substituting $g_{j i}(\mathbf{x}, t)$ in Eq. (52):

$$
\begin{aligned}
g_{j i}\left(\mathbf{x}+c_{j i} \delta t, t+\delta t\right)= & g_{j i}(\mathbf{x}, t)-\frac{\omega}{\tau_{1 j}}\left[g_{j i}(\mathbf{x}, t)-f^{*}(\mathbf{x}, f, t)\right] \\
& -\frac{\omega}{\tau_{2 j}}\left[f_{j i}^{*}(\mathbf{x}, f, t)-f_{j i}^{\mathrm{eq}}(\mathbf{x}, f, t)\right]-\omega F_{j i},
\end{aligned}
$$

with $\omega=2 \delta t \tau_{1 j} /\left(2 \tau_{1 j}+\delta t\right)$. The term $F_{j i}$ in Eq. (54) accounts for the velocity correction $F_{V j i \alpha}$ that has to be applied to both models (mixture-averaged correction velocity), and the pressure correction $F_{P j i \alpha \beta}$ for model II.

The auxiliary function $g_{j i}(t)$ depends on $f_{j i}^{*}(t, f)$ and $f_{j i}^{\mathrm{eq}}(t, f)$ that require the evaluation of the moments of $f_{j i}(t)$. For both models these moments can be computed from Eq. (53). For the first collision model (model I):

$$
\begin{gathered}
\rho_{j}(f)=\rho_{j}(g), \\
J_{j \alpha}(f)=\frac{J_{j \alpha}(g)+\frac{\delta t}{2 \tau_{1 j}^{\mathrm{I}}} \frac{\rho_{j}}{\rho} J_{\alpha}(g)+\frac{\delta t}{2} \sum_{i} F_{j i}}{1+\frac{\delta t}{2 \tau_{1 j}^{\mathrm{I}}}}, \\
J_{\alpha}(f)=J_{\alpha}(g) .
\end{gathered}
$$

Equations (55) can be used for model II by substituting $\tau_{1 j}^{\mathrm{I}}$ with $\tau_{2 j}^{\mathrm{II}}$. Model I requires two more equations for the pressure evaluation

$$
\begin{gathered}
P_{j \alpha \beta}(f)=\frac{P_{j \alpha \beta}(g)+\frac{\delta t}{2 \tau_{2}^{\mathrm{I}}} P_{j \alpha \beta}(g)^{\mathrm{eq}}}{1+\frac{\delta t}{2 \tau_{2}^{\mathrm{I}}}}, \\
P_{j \alpha \beta}^{\mathrm{eq}}(f)=P_{j \alpha \beta}^{\mathrm{eq}}(g) .
\end{gathered}
$$

The nonconserved moments of each individual species are different for the two distribution functions $g$ and $f$. These transformations have to be taken into account whenever a nonconserved moment is needed [47] [for example, in the evaluation of $f^{*}(f)$ or in computing the forcing terms in Eqs. (34) and (46)].

The discretization scheme transforms the initial implicit time integration problem in $f$ into an explicit expression in $g$. Moreover, it should be emphasized that the time integration scheme is second-order accurate.

Since in a mixture the masses of the species are generally unequal, the species lattice speed will be different (Fig. 1). The time step is defined as $d t_{j}=d x_{j} / c_{j}$, where $d x_{j}$ is the lattice spacing of component $j$. In order to have the same time step for all species, different lattice grids (one for each species) have to be used, and the corresponding lattice spacing ratio is related to the ratio of the lattice speeds of the species, i.e., to the inverse of the square root of the molecular weight ratio. This implies that the heavy species populations will reside on a finer grid or, equivalently, that the light species in one time step are diffusing faster than the heavier (Graham's effusion law). The spacial discretization is implemented as suggested in Ref. [2] with one substantial difference: instead of using the Lagrange interpolation, the second-order volumetric interpolation suggested by [48] is preferred, since this scheme ensures locally density and momentum conservation and, at the same time, allows for an easy implementation of the wall boundaries.

\section{APPLICATIONS}

Two applications are presented, a macroscopic and a microscopic one. In the former case, the model is validated against a continuum model and in the latter against DSMC and kinetic theory.

\section{A. Opposing jets}

The model is first tested in the simulation of two planar opposing jets, issuing mixtures with different concentrations (Fig. 2). Yamamoto et al. $[49,50]$ have already studied such a flow with a LB formulation using the single-fluid approach with constant diffusion coefficients and within the constant density approximation (i.e. the flow was fully decoupled from the species and temperature equations) to simulate premixed combustion in a nonisothermal flow.

The simulation domain is discretized with $200 \times 400$ nodes. Along the vertical boundaries $\left(x= \pm L_{x} / 2\right)$ inlet conditions are applied at $-0.4 L_{x} \leqslant y \leqslant 0.4 L_{x}$ by imposing the incoming populations to be equal to the equilibrium populations corresponding to the inlet velocity and concentration profiles. The inlet velocities at $-0.4 L_{x} \leqslant y \leqslant 0.4 L_{x}$ are set 


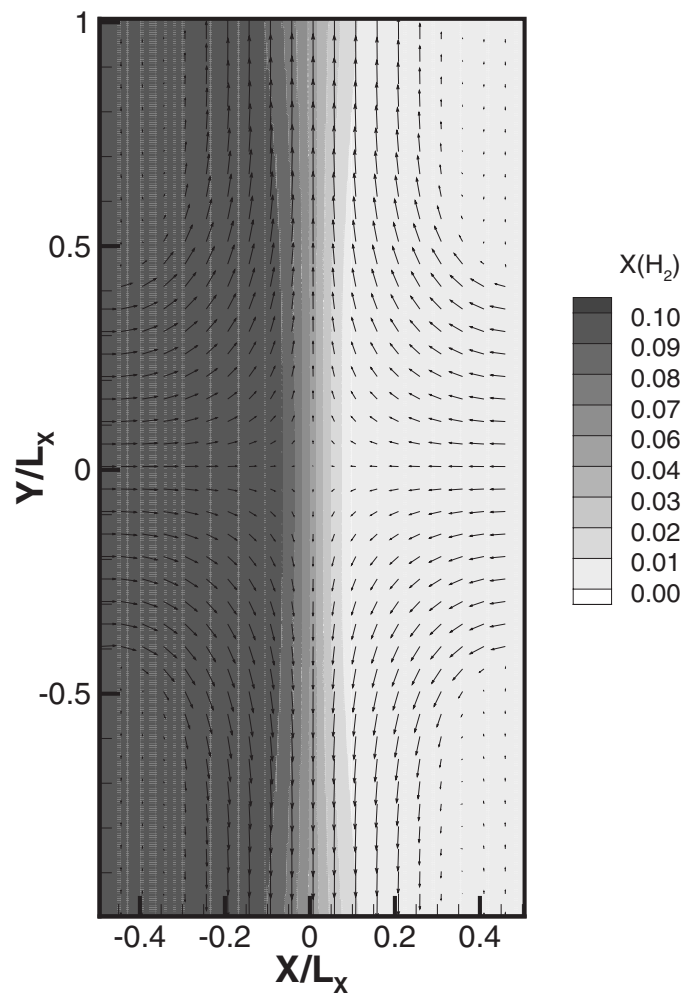

FIG. 2. LB simulation of the mixing in an opposed-jet configuration: Velocity vector plot superimposed on the $\mathrm{H}_{2}$ mole fraction isocontours.

such that the two inlet streams have equal momenta, resulting in a stagnation plane located halfway between the two nozzles. The molar fractions at the two nozzles are

$$
\left.\begin{array}{rl}
X\left(\mathrm{H}_{2}\right) & =0.10, \\
X\left(\mathrm{~N}_{2}\right) & =0.85, \\
X\left(\mathrm{O}_{2}\right) & =0.00, \\
X\left(\mathrm{H}_{2} \mathrm{O}\right) & =0.05,
\end{array}\right\} x=-\frac{L}{2},
$$

In the remaining vertical boundaries, reflective boundary conditions are imposed. The incoming populations along the horizontal upper and lower boundaries are extrapolated from the previous node.

The binary diffusion coefficients $\mathcal{D}_{j k}$ and the viscosity $\mu_{j}$ are evaluated at each time step locally as a function of the local concentrations, pressure, and temperature (the reference temperature $T_{0}$ is fixed to $300 \mathrm{~K}$ ) using the CHEMKIN transport properties package [38]. Once $\mu_{j}$ and $\mathcal{D}_{j k}$ are computed, it is possible to evaluate the two local relaxation times $\tau_{1 j}$ [Eq. (28)] and $\tau_{2 j}$ [Eq. (42)]. When $\tau_{1 j}<\tau_{2 j}$ model I is used

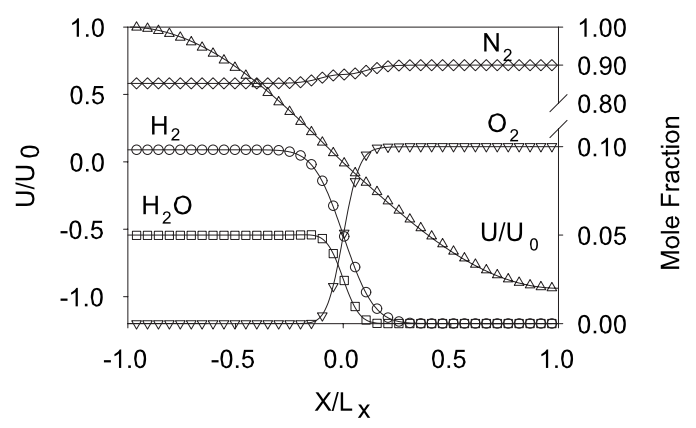

FIG. 3. Opposed-jet mixing: Comparison of the velocity and the concentrations along the plane of symmetry $(y=0)$ between the LB mixture model (symbols) and the 1D continuum code OPPDIF predictions (continuous lines).

such that $\tau_{1 j}^{\mathrm{I}}=\tau_{1 j}$ and $\tau_{2 j}^{\mathrm{I}}=\tau_{2 j}$. When $\tau_{1 j}>\tau_{2 j}$ model II is employed, with $\tau_{1 j}^{\mathrm{II}}=\tau_{2 j}$ and $\tau_{2 j}^{\mathrm{II}}=\tau_{1 j}$. It is noted that at a given grid point different species may require different models.

Figure 3 compares the results of the LB computations with those of the macroscopic 1D code OPPDIF [51] (the counterflow geometry reduces to a $1 \mathrm{D}$ problem along the symmetry plane) using the mixture-averaged approach. A good agreement is found for both the velocity and the concentration profiles. This is a severe test for the code since the species can diffuse against the bulk flow velocity. Moreover, the code is stable, even though the mole fraction of one or more components drops eventually to zero $\left(10^{-14}\right.$ was the minimum allowed concentration) in some regions.

\section{B. Micro-Couette flow}

The flows of mixtures in microchannels is a topic of practical interest. However, due to the complexity of the problem and the considerable computational effort the pertinent literature is scarce. In order to validate the present model also in the microscopic scale, the slip coefficient of a binary mixture in a planar Couette flow is compared against recent DSMC calculations [52] and against the linearized McCormack kinetic model of the Boltzmann equation solved by the discrete velocity method at steady state [53]. The binary slip coefficient $\sigma_{12}$ is defined as

$$
U_{0}-U_{w}=\sigma_{12} \lambda\left(\frac{\partial U}{\partial y}\right)_{y=0},
$$

where $U_{0}$ and $U_{w}$ are the velocity of the fluid at the wall $(y=0)$ and the velocity of the wall, respectively, while $\lambda$ is the mean free path

$$
\lambda=\frac{\mu v}{P}, \quad v=\left(\frac{3 R T}{X_{1} m_{1}+X_{2} m_{2}}\right)^{1 / 2},
$$

where $v$ is the most probable velocity of the mixture. It is noted that in Eq. (60) within the LB framework $v$ is defined with a factor of 3 [18] and not with the factor 2 used in the continuum 2D case [52,53]. In Ref. [18], an exact solution of the nonlinear LB kinetic equations for the stationary Couette flow at nonvanishing $\mathrm{Kn}$ was established in the case of single 

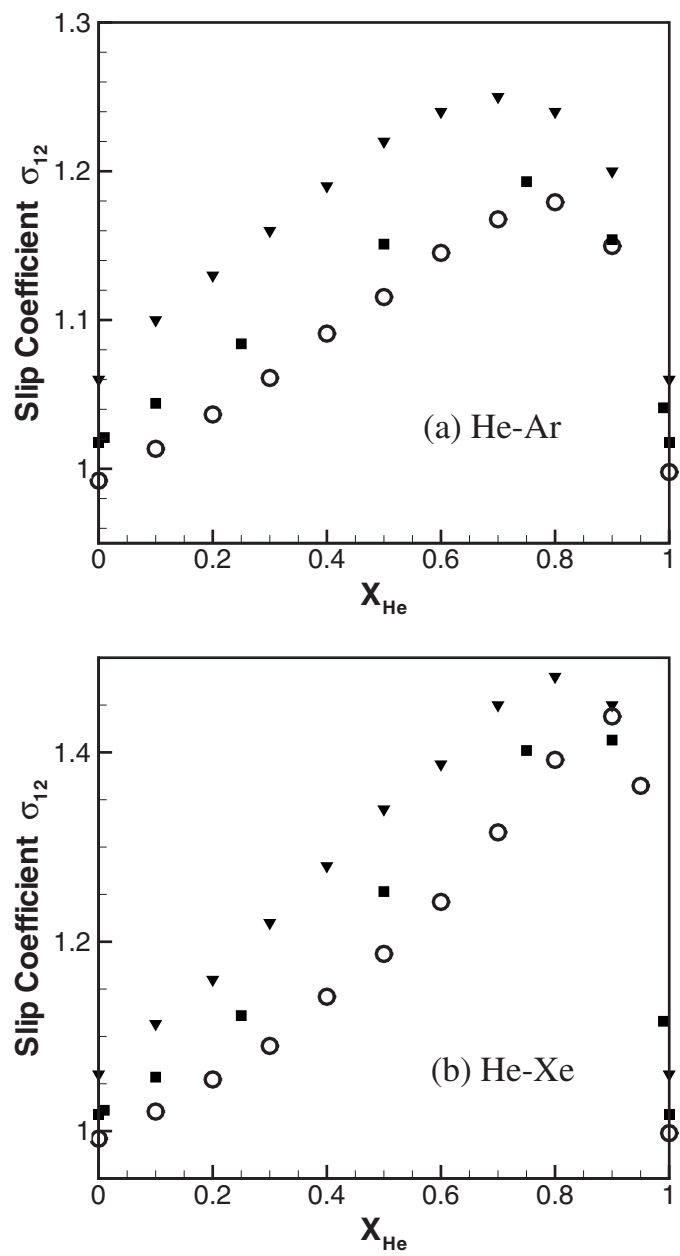

FIG. 4. Slip coefficient versus helium molar fraction $X_{\mathrm{He}}$. (a) Helium-argon mixture. (b) Helium-xenon mixture. Squares: linearized kinetic theory results from Ref. [53]. Triangles: DSMC results [52]. Circles: present work.

component. This solution can be used in order to validate the slip coefficient for the present model in the limiting case of single component flows. From Ref. [18] it follows that $\sigma_{11}$ $=1$. This value can serve as an indicator of the accuracy of the implementation.

Simulations are performed at Knudsen number $\mathrm{Kn}=0.2$ and at different mole fractions $X_{\mathrm{He}}$ of the light component (helium). The top (bottom) wall is moving with the velocity $U_{w}\left(-U_{w}\right)$ and diffusive wall boundary conditions [20] are applied at the plates. Periodic boundary conditions are applied in the flow direction. The resulting slip coefficient is shown in Fig. 4 for two different mixtures, helium-argon [Fig. 4(a)] and helium-xenon [Fig. 4(b)]. In accordance with previous studies [52,53], mixing increases the slip coefficient which becomes larger compared to the corresponding singlecomponent case. The difference is more pronounced in the He-Xe case due to the larger mass ratio (32.75). The curves in Fig. 4 are not symmetric, but show a maximum shifted toward the larger concentration of the light component. LB calculations predict for both mixtures the correct behavior of the slip coefficient. In addition, the concentration corresponding to the maximum of the slip curve is in good agreement with the kinetic theory calculations (around 0.8 for the mixture $\mathrm{He}-\mathrm{Ar}$ and around 0.9 for $\mathrm{He}-\mathrm{Xe}$ ). The DSMC predict a somewhat smaller value for the location of the maximum. The difference between the kinetic theory curves and the LB model can be attributed to the assumption of the viscosity dependence on the concentration [Eq. (40)]. Regarding the accuracy of the implementation, the code shows for the case $X_{\mathrm{He}}=1$ an error $\epsilon=0.2 \%$ compared to the exact solution [18], while for $X_{\mathrm{He}}=0, \epsilon=0.7 \%$ for both $\mathrm{Ar}$ and Xe. Finally, it should be noted that the current transient LB simulations require a few minutes while the corresponding CPU times in DSMC are of the order of days [52]. Moreover, the present model allows for transient simulations of multicomponent systems with complex boundaries with practically the same computational effort (the code computational cost scales linearly with the number of species).

\section{CONCLUSIONS}

A lattice Boltzmann model for the simulation of multicomponent isothermal mixtures was developed and tested. The implementation consists of two complementary models and the choice of the appropriate model depends on the local Schmidt number of species $j$. Within the mixture-averaged approximation, the Stefan-Maxwell diffusion equation is recovered. The model is applied to simulate the mixing in opposing planar jets. Along the plane of symmetry, results of the LB simulation are in a good agreement with a onedimensional finite-difference macroscopic solver. The aptness of the LB model in microscale flows is shown in the second test case by comparing the slip coefficients obtained from LB simulations of a mixture in a microchannel with DSMC and linearized kinetic theory. Results are in a good agreement in terms of slip coefficient shapes and location of maximum slip coefficient. The model is currently extended to take into account surface chemical reactions in nonisothermal flows.

\section{ACKNOWLEDGMENTS}

I.V.K. greatly acknowledges support by the BFE-Project No. 100862 and very useful discussions of results with Professor M. S. Ivanov and Academician A. K. Rebrov. S.A. and I.V.K. acknowledge the support from the Competence Center of Energy and Mobility (CCEM) of Switzerland.

\section{APPENDIX: LAGRANGE MULTIPLIERS}

The expressions for Lagrange multipliers introduced in Eq. (9) read as follows: 


$$
\begin{aligned}
& \chi=3\left\{P_{y y}\left[2-6 v_{y}^{2}+18 v_{y}^{4}+27 v_{x}^{6}\left(-1+9 v_{y}^{2}\right)+27 v_{x}^{4}\left(1-14 v_{y}^{2}+9 v_{y}^{4}\right)-9 v_{x}^{2}\left(1-8 v_{y}^{2}+12 v_{y}^{4}\right)\right]+3 P_{x y} v_{x} v_{y}\left[9 v_{x}^{4}\left(-4+9 v_{y}^{2}\right)-4(1\right.\right. \\
& \left.\left.-3 v_{y}^{2}+9 v_{y}^{4}\right)+3 v_{x}^{2}\left(4+3 v_{y}^{2}+27 v_{y}^{4}\right)\right]+P_{x x}\left[2-9 v_{y}^{2}+27 v_{y}^{4}-27 v_{y}^{6}+9 v_{x}^{4}\left(2-12 v_{y}^{2}+27 v_{y}^{4}\right)+3 v_{x}^{2}\left(-2+24 v_{y}^{2}-126 v_{y}^{4}\right.\right. \\
& \left.\left.\left.+81 v_{y}^{6}\right)\right]\right\} \Delta^{-1} \text {, } \\
& \beta_{x}=\left\{P_{y y}\left[-54 v_{x} v_{y}^{2}-243 v_{x}^{3} v_{y}^{2}-486 v_{x} v_{y}^{4}+486 v_{x}^{5} v_{y}^{2}+3159 v_{x}^{3} v_{y}^{4}+486 v_{x} v_{y}^{6}-2187 v_{x}^{5} v_{y}^{4}-2187 v_{x}^{3} v_{y}^{6}\right]+P_{x x}\left[+36 v_{x}-54 v_{x}^{3}\right.\right. \\
& \left.-162 v_{x} v_{y}^{2}+729 v_{x}^{3} v_{y}^{2}+486 v_{x} v_{y}^{4}-486 v_{x} v_{y}^{6}-486 v_{x}^{5} v_{y}^{2}-3159 v_{x}^{3} v_{y}^{4}+2187 v_{x}^{5} v_{y}^{4}+2187 v_{x}^{3} v_{y}^{6}\right]+P_{x y}\left[+36 v_{y}-162 v_{y}^{3}-270 v_{x}^{2} v_{y}\right. \\
& \left.\left.+486 v_{y}^{5}+1215 v_{x}^{2} v_{y}^{3}-486 v_{y}^{7}+486 v_{x}^{6} v_{y}-2673 v_{x}^{2} v_{y}^{5}+486 v_{x}^{4} v_{y}^{3}+2187 v_{x}^{2} v_{y}^{7}-2187 v_{x}^{6} v_{y}^{3}\right]\right\} \Delta^{-1} \text {, } \\
& \beta_{y}=\left\{P_{x x}\left[-2187 v_{y}^{3} v_{x}^{6}+486 v_{y} v_{x}^{6}-2187 v_{y}^{5} v_{x}^{4}+3159 v_{y}^{3} v_{x}^{4}-486 v_{y} v_{x}^{4}+486 v_{y}^{5} v_{x}^{2}-243 v_{y}^{3} v_{x}^{2}-54 v_{y} v_{x}^{2}\right]+P_{y y}\left[2187 v_{y}^{3} v_{x}^{6}-486 v_{y} v_{x}^{6}\right.\right. \\
& \left.+2187 v_{y}^{5} v_{x}^{4}-3159 v_{y}^{3} v_{x}^{4}+486 v_{y} v_{x}^{4}-486 v_{y}^{5} v_{x}^{2}+729 v_{y}^{3} v_{x}^{2}-162 v_{y} v_{x}^{2}-54 v_{y}^{3}+36 v_{y}\right]+P_{x y}\left[2187 v_{y}^{2} v_{x}^{7}-486 v_{x}^{7}-2673 v_{y}^{2} v_{x}^{5}\right. \\
& \left.\left.+486 v_{x}^{5}-2187 v_{y}^{6} v_{x}^{3}+486 v_{y}^{4} v_{x}^{3}+1215 v_{y}^{2} v_{x}^{3}-162 v_{x}^{3}+486 v_{y}^{6} v_{x}-270 v_{y}^{2} v_{x}+36 v_{x}\right]\right\} \Delta^{-1} \text {, } \\
& \gamma_{x x}=\left\{P_{y y}\left[-243 v_{y}^{2} v_{x}^{4}-243 v_{y}^{4} v_{x}^{2}+243 v_{y}^{2} v_{x}^{2}\right]+P_{x x}\left(-2187 v_{x}^{2} v_{y}^{6}+243 v_{y}^{6}-2187 v_{x}^{4} v_{y}^{4}+2916 v_{x}^{2} v_{y}^{4}-243 v_{y}^{4}+486 v_{x}^{4} v_{y}^{2}-486 v_{x}^{2} v_{y}^{2}\right.\right. \\
& \left.\left.+81 v_{y}^{2}-18\right)+P_{x y}\left[2187 v_{y}^{3} v_{x}^{5}-486 v_{y} v_{x}^{5}+2187 v_{y}^{5} v_{x}^{3}-2673 v_{y}^{3} v_{x}^{3}+486 v_{y} v_{x}^{3}\right]\right\} \Delta^{-1}, \\
& \gamma_{y y}=\left\{P_{x x}\left[-243 v_{y}^{2} v_{x}^{4}-243 v_{y}^{4} v_{x}^{2}+243 v_{y}^{2} v_{x}^{2}\right]+P_{y y}\left[-2187 v_{y}^{2} v_{x}^{6}+243 v_{x}^{6}-2187 v_{y}^{4} v_{x}^{4}+2916 v_{y}^{2} v_{x}^{4}-243 v_{x}^{4}+486 v_{y}^{4} v_{x}^{2}-486 v_{y}^{2} v_{x}^{2}\right.\right. \\
& \left.\left.+81 v_{x}^{2}-18\right]+P_{x y}\left[2187 v_{y}^{3} v_{x}^{5}+2187 v_{y}^{5} v_{x}^{3}-2673 v_{y}^{3} v_{x}^{3}-486 v_{y}^{5} v_{x}+486 v_{y}^{3} v_{x}\right]\right\} \Delta^{-1}, \\
& \gamma_{x y}=\left\{P_{x x}\left[2187 v_{y}^{3} v_{x}^{5}-486 v_{y} v_{x}^{5}+2187 v_{y}^{5} v_{x}^{3}-2673 v_{y}^{3} v_{x}^{3}+486 v_{y} v_{x}^{3}\right]+P_{y y}\left[2187 v_{y}^{3} v_{x}^{5}+2187 v_{y}^{5} v_{x}^{3}-2673 v_{y}^{3} v_{x}^{3}-486 v_{y}^{5} v_{x}\right.\right. \\
& \left.+486 v_{y}^{3} v_{x}\right]+P_{x y}\left[-2187 v_{y}^{2} v_{x}^{6}+486 v_{x}^{6}-4374 v_{y}^{4} v_{x}^{4}+2673 v_{y}^{2} v_{x}^{4}-486 v_{x}^{4}-2187 v_{y}^{6} v_{x}^{2}+2673 v_{y}^{4} v_{x}^{2}-729 v_{y}^{2} v_{x}^{2}+162 v_{x}^{2}+486 v_{y}^{6}\right. \\
& \left.\left.-486 v_{y}^{4}+162 v_{y}^{2}-36\right]\right\} \Delta^{-1}
\end{aligned}
$$

where $\Delta$ is defined as

$$
\Delta=\rho\left[-4+18 v_{y}^{2}-54 v_{y}^{4}+54 v_{y}^{6}+27 v_{x}^{6}\left(2-27 v_{y}^{2}+81 v_{y}^{4}\right)+27 v_{x}^{4}\left(-2+33 v_{y}^{2}-135 v_{y}^{4}+81 v_{y}^{6}\right)-9 v_{x}^{2}\left(-2+21 v_{y}^{2}-99 v_{y}^{4}+81 v_{y}^{6}\right)\right] .
$$

[1] L. S. Luo and S. S. Girimaji, Phys. Rev. E 67, 036302 (2003).

[2] M. E. McCracken and J. Abraham, Phys. Rev. E 71, 046704 (2005).

[3] P. Asinari, Phys. Fluids 17, 067102 (2005).

[4] P. Asinari, Phys. Rev. E 73, 056705 (2006).

[5] E. G. Flekkoy, Phys. Rev. E 47, 4247 (1993).

[6] Z. Guo and T. S. Zhao, Phys. Rev. E 68, 035302(R) (2003).

[7] A. Lamura, G. Gonnella, and J. M. Yeomans, Europhys. Lett. 45, 314 (1999).

[8] E. Orlandini, M. R. Swift, and J. M. Yeomans, Europhys. Lett. 32, 463 (1995).

[9] W. R. Osborn, E. Orlandini, M. R. Swift, J. M. Yeomans, and J. R. Banavar, Phys. Rev. Lett. 75, 4031 (1995).

[10] X. W. Shan and H. D. Chen, Phys. Rev. E 47, 1815 (1993).

[11] X. W. Shan and G. Doolen, J. Stat. Phys. 81, 379 (1995).

[12] X. W. Shan and G. Doolen, Phys. Rev. E 54, 3614 (1996).

[13] X. W. Shan and X. Y. He, Phys. Rev. Lett. 80, 65 (1998).

[14] V. Sofonea and R. F. Sekerka, Physica A 299, 494 (2001).

[15] M. R. Swift, E. Orlandini, W. R. Osborn, and J. M. Yeomans, Phys. Rev. E 54, 5041 (1996).

[16] Q. Li and A. J. Wagner, Phys. Rev. E 76, 036701 (2007).
[17] I. Halliday, A. P. Hollis, and C. M. Care, Phys. Rev. E 76, 026708 (2007).

[18] S. Ansumali, I. V. Karlin, S. Arcidiacono, A. Abbas, and N. Prasianakis, Phys. Rev. Lett. 98, 124502 (2007).

[19] S. Ansumali, I. V. Karlin, C. E. Frouzakis, and K. B. Boulouchos, Physica A 359, 289 (2006).

[20] S. Ansumali and I. V. Karlin, Phys. Rev. E 66, 026311 (2002).

[21] R. Benzi, L. Biferale, M. Sbragaglia, S. Succi, and F. Toschi, Math. Comput. Simul. 72, 84 (2006).

[22] R. Benzi, L. Biferale, M. Sbragaglia, S. Succi, and F. Toschi, Europhys. Lett. 74, 651 (2006).

[23] A. Gabrielli, S. Succi, and E. Kaxiras, Comput. Phys. Commun. 147, 516 (2002).

[24] C. Y. Lim, C. Shu, X. D. Niu, and Y. T. Chew, Phys. Fluids 14, 2299 (2002).

[25] X. B. Nie, G. D. Doolen, and S. Y. Chen, J. Stat. Phys. 107, 279 (2002).

[26] X. D. Niu, C. Shu, and Y. T. Chew, Europhys. Lett. 67, 600 (2004)

[27] X. D. Niu, C. Shu, and Y. T. Chew, Int. J. Mod. Phys. C 16, 1927 (2005). 
[28] C. Shu, X. D. Niu, and Y. T. Chew, J. Stat. Phys. 121, 239 (2005).

[29] V. Sofonea and R. F. Sekerka, J. Comput. Phys. 207, 639 (2005).

[30] S. Succi, A. Gabrielli, G. Smith, and E. Kaxiras, Eur. Phys. J.: Appl. Phys. 16, 71 (2001).

[31] S. Succi, Phys. Rev. Lett. 89, 064502 (2002).

[32] Z. W. Tian, C. Zou, Z. H. Liu, Z. L. Guo, H. J. Liu, and C. G. Zheng, Int. J. Mod. Phys. C 17, 603 (2006).

[33] Y. Zhou, R. Zhang, I. Staroselsky, H. Chen, W. T. Kim, and M. S. Jhon, Physica A 362, 68 (2006).

[34] S. Arcidiacono, S. Ansumali, I. V. Karlin, J. Mantzaras, and K. B. Boulouchos, Math. Comput. Simul. 72, 79 (2006).

[35] S. Arcidiacono, J. Mantzaras, S. Ansumali, I. V. Karlin, C. Frouzakis, and K. B. Boulouchos, Phys. Rev. E 74, 056707 (2006).

[36] R. B. Bird, W. E. Stewart, and E. N. Lightfoot, Transport Phenomena (Wiley, New York, 1960).

[37] F. Williams, Combustion Theory (Benjamin/Cumming, California, 1986).

[38] R. J. Kee, G. Dixon-Lewis, J. Warnatz, M. E. Coltrin, and J. A. Miller, A Fortran Computer Code Package for the Evaluation of Gas-Phase Multicomponent Transport Properties (Sandia National Laboratories, Sandia, 1996).

[39] A. N. Gorban and I. V. Karlin, Physica A 206, 401 (1994).

[40] C. D. Levermore, J. Stat. Phys. 83, 1021 (1996).
[41] I. V. Karlin, A. Ferrante, and H. C. Ottinger, Europhys. Lett. 47, 182 (1999).

[42] S. Ansumali, I. V. Karlin, and H. C. Öttinger, Europhys. Lett. 63, 798 (2003).

[43] A. N. Gorban and I. V. Karlin, Invariant Manifolds for Physical and Chemical Kinetics (Springer, Berlin, 2005).

[44] A. N. Gorban and I. V. Karlin, Physica A 360, 325 (2006).

[45] S. S. Chikatamarla, S. Ansumali, and I. V. Karlin, Europhys. Lett. 74, 215 (2006).

[46] C. Wilke, J. Chem. Phys. 18, 517 (1950).

[47] S. Ansumali, S. Arcidiacono, S. Chikatamarla, N. Prasianakis, A. N. Gorban, and I. V. Karlin, Eur. Phys. J. B 56, 135 (2007).

[48] H. Chen, Phys. Rev. E 58, 3955 (1998).

[49] K. Yamamoto, X. He, and G. D. Doolen, J. Stat. Phys. 107, 367 (2002).

[50] K. Yamamoto, X. He, and G. D. Doolen, JSME Int. J., Ser. B 47, 403 (2004).

[51] A. E. Lutz, R. J. Kee, J. F. Grcar, and F. M. Rupley, OPPDIF: A Fortran Program for Computing Opposed-Flow Diffusion Flames (Sandia National Laboratories, Sandia, 1997).

[52] T. Hyakutake, K. Yamamoto, and H. Takeuchi, in Rarefied Gas Dynamics: 24th International Symposium on Rarefied Gas Dynamics, edited by Mario Capitelli, AIP Conf. Proc. No. 762 (AIP, Melville, N.Y., 2005), pp. 780-788.

[53] F. Sharipov and D. Kalempa, Phys. Fluids 15, 1800 (2003). 